International Journal of Stem Cell Research and Transplantation (IJST)

ISSN 2328-3548

\title{
Investigating Cell Surface Markers and Differentiation Potential of Compact Bone-Derived Mesenchymal Stem Cells
}

Mitra $\mathrm{NK}^{1 *}$, Tan $\mathrm{CY}^{2}$, Bindal $\mathrm{U}^{1}$, Chua CLL ${ }^{3}$, Wong EH ${ }^{1}$, Parhar $\mathrm{IS}^{4}$, Soga $\mathrm{T}^{4}$

${ }^{1}$ School of Medicine, Taylor's University Lakeside Campus, Selangor, Malaysia.

${ }^{2}$ Research Laboratory, School of Medicine, Pharmacy and Biosciences, Taylor's University Lakeside Campus, Selangor, Malaysia.

${ }^{3}$ School of Biosciences, Taylor's University Lakeside Campus, Selangor, Malaysia.

${ }^{4}$ Brain Research Institute, School of Medicine and Health Sciences, Monash University Sunway Campus, Selangor, Malaysia.

\begin{abstract}
Background: The differentiation potential of mesenchymal stem cells (MSCs) derived from the bone-tissue to multiple lineages is not clear.

Objective: This study was conducted to investigate the surface antigen expression and multilineage stem cell potential of the cells derived from culture of collagenase digested marrow-free compact bones of C57BL/6 mouse.

Materials \& Methods: Long bones of C57BL/6 mouse $(n=6)$ were collected aseptically and bone marrow was flushed out. Collagenase-digested bone fragments were washed and cultured in plastic flasks. The plastic-adherent fibroblast-like spindle-shaped cells were cultured sequentially in multiple passages in low-glucose DMEM (Dulbecco's Modified Eagle's Medium) supplemented with $15 \% \mathrm{FBS}$ (Foetal Bovine Serum) and antibiotics in a $37^{\circ} \mathrm{C}$ incubator with $5 \% \mathrm{CO}_{2}$. Immunophenotyping for cell surface markers was done using flow cytometry. The cells were differentiated into the osteoblastic, adipogenic and chondrogenic lineages.

Results: The culture of the adherent cells exhibited active proliferation and multiplication in consequent passages. The cultured cells revealed evidence of adipogenic and osteogenic differentiation confirmed by staining with oil red $\mathrm{O}$ and von Kossa stains. Under flow cytometry observation, a significant proportion of cultured cells expressed CD29 and stem cell antigen (Sca-1). Only 9.8\% cells showed expression of CD105. These MSCs exhibited low ability in chondrogenic differentiation, which can potentially be attributed to their lack of CD105 expression. Lack of expression of CD45 showed evidence of absence of hematopoietic stem cells.

Conclusion: This study showed that murine compact bone-chip culture can yield MSCs with significant proliferation capacity. The cells displayed the ability to differentiate into osteoblast and adipocyte lineages.
\end{abstract}

Keywords: Mesenchymal Stem Cells; Mouse; Compact Bone; Adipogenesis; Osteogenesis; Chondrogenesis.

\section{*Corresponding Author:}

Dr. Nilesh Kumar Mitra,

Associate Professor, School of Medicine, Taylor's University Lakeside Campus, No.1 Jalan Taylors, Subang Jaya, 47500, Selnagor, Malaysia.

Tel: $+603-56295495$

Fax: +603-56295455

Email: NileshKumar.Mitra@taylors.edu.my

Received: March 25, 2015

Accepted: April 17, 2015

Published: April 20, 2015

Citation: Mitra NK, et al., (2015) Investigating Cell Surface Markers and Differentiation Potential of Compact Bone-Derived Mesenchymal Stem Cells. Int J Stem Cell Res Transplant 03(1), 91-95. doi: http:/ / dx.doi. org $/ 10.19070 / 2328-3548-1500015$

Copyright: Mitra NK ${ }^{\circ}$ 2015. This is an open-access article distributed under the terms of the Creative Commons Attribution License, which permits unrestricted use, distribution and reproduction in any medium, provided the original author and source are credited.

\section{Introduction}

Mesenchymal stem cells (MSCs) were originally isolated from the yellow bone marrow located in the medullary cavity of long bones of mouse by Friedenstein et al. [1]. Consequent studies confirmed that these cells could also be isolated from the human bone marrow and have the potential to differentiate into cells with mesenchymal lineages such as adipocytes, chondrocytes, myoblasts and osteoblasts [2]. These cells are capable of proliferation, self-renewal and regeneration into tissues of mesenchymal lineage. MSCs from bone marrow have been isolated from different species including mouse, rat, rabbit, monkey and human [3-7]. Although the cells share several characteristics, variations occur among the species in the expression of antigens [8]. Among the various methods of the isolation of MSCs (plastic adherence, density gradient centrifugation and immunomagnetic selection), plastic adherence is an easy and popular method [9-11]. Plastic adherence failed to yield relatively homogeneous MSCs population from the murine bone marrow because of the hematopoietic cell 
contamination [12]. Separation of the hematopoietic stem cells from the bone marrow-derived MSCs requires multiple passaging of the culture.

Isolation and culture of mouse MSCs from the compact bone was proposed to be easier compared to the similar culture from the bone marrow $[13,14]$. Explant culture from the trabecular bone pieces showed the presence of MSCs that could differentiate into osteoblasts, adipocytes and chondrocytes. These cells also expressed surface antigens similar to the bone marrow-derived MSCs, such as lacking the hematopoietic and common leukocyte markers (CD45), and expressing the markers related to adhesion (CD29) and stem cells (CD105) [15]. However it was reported that compared to the bone marrow-derived MSCs, compact bone-derived MSCs showed reduced capacity for multi-lineage differentiation [15]. In this study, we isolated, expanded and characterized MSCs by the primary culture of compact bone chips from C57BL/ 6 mouse using standard method of plastic adherence to test the differentiation potential and expression of surface antigens.

\section{Materials and Methods}

\section{Mice}

Four-week-old female C57BL/ 6 mice $(n=6)$ were maintained at the animal facility of the university. All mice were housed under specific pathogen-free conditions. The procedures on the mice were approved by the Animal Care and Use Committee of the university.

\section{Isolation of plastic adherent MSCs from culture of compact bone}

The C57BL/ 6 mice were sacrificed under anesthesia followed by cervical dislocation. Femur, tibia and humerus bones were cut in between the upper and lower epiphyses and collected under aseptic condition. The bones were separated from the muscles and soft tissues by gentle abrasion using sterile gauze pieces. Bone marrow was flushed out by injecting Hank's balanced salt solution into the medullary cavity, until the bone pieces became pale. The marrow-free bones were then chopped into minute pieces using the micro-dissecting scissors. Dulbecco's Modified Eagle Medium (DMEM) which contained $1000 \mathrm{mg} / \mathrm{L}$ glucose, $584 \mathrm{mg} / \mathrm{L}$ L-Glutamine and, was supplemented with 15\% MSC qualified Fetal Bovine Serum (FBS), $100 \mathrm{IU} / \mathrm{mL}$ Penicillin and $100 \mu \mathrm{g} / \mathrm{mL}$ Streptomycin (all from GIBCO), was used to store the bones and for subsequent cultures. The bone pieces were digested in a mixture of DMEM and Collagenase II (GIBCO) $\left(1 \mathrm{mg} \mathrm{m}^{-1}\right.$ weight/ volume) for 2 hours in a shaking incubator $\left(37^{\circ} \mathrm{C}, 200 \mathrm{rpm}\right)$. This resulted in the formation of a coagulum because of the adherence of the bony pieces with each other. The medium was aspirated and discarded. The bone chips were washed three times with the DMEM. The bone chips were then plated in a $25 \mathrm{~cm}^{2}$ plastic culture flask in low glucose DMEM supplemented with FBS and antibiotics, in proportion, as described above, and incubated at 37 ${ }^{\circ} \mathrm{C}$ in $5 \% \mathrm{CO}_{2}$. On the third day, the non-adherent circular floating cells were removed by a complete media change. After seven days, the adherent MSCs were harvested by trypsinization with $0.25 \%$ trypsin containing $1 \mathrm{mM}$ EDTA (GIBCO). The cell count was done using the Trypan blue exclusion assay and plating was done at variable densities ranging from 1600 to 2500 cells $/ \mathrm{cm}^{2}$.
The adherent cells were cultured in consecutive passages once per week at a split ratio of 1:2 until the $12^{\text {th }}$ passage. The variation in the cell count in consecutive passages was monitored.

\section{Differentiation of plastic adherent MSCs}

Differentiation to adipocyte: The MSCs obtained based on plastic adherence from bone chips culture in passage-4 were plated at a density of $1 \times 10^{4}$ cells in Mesencult proliferation media (Stemcell Technologies, Canada) in each well of a 6-well plate. The culture was maintained for seven days or until the adherent layer of at least $80 \%$ confluence was formed. The media was aspirated and replaced with Mesencult basal medium containing 20\% Mesencult adipogenic stimulatory supplement (Stemcell Technologies, Canada). The medium was replenished once every three days for three weeks. The adipogenic stimulatory supplement contained dexamethasone, indomethacin and insulin. Adipocytes differentiated from MSCs were fixed with 4\% paraformaldehyde, washed with $60 \%$ isopropanol and then stained with oil red $\mathrm{O}$ followed by counterstaining of nuclei by haematoxylin.

Differentiation to osteoblast: Passage-4 MSCs were plated in proliferation media in a 6 -well plate as described above in adipocyte differentiation. When the cultured cells were $80 \%$ confluent, the proliferation media was replaced with Mesencult basal medium containing 25\% Mesencult osteogenic stimulatory supplement (Stemcell Technologies, Canada). The osteogenic stimulatory supplement contained dexamethasone, $\beta$-glycerophosphate and ascorbic acid. The medium was replenished once every three days for three weeks. The brownish-black deposits at the bottom of the plate indicated appearance of calcium salts, which were further identified using von Kossa staining. The differentiated cells were fixed with $4 \%$ paraformaldehyde, incubated with $1 \%$ silver nitrate for 2 hours followed by removal of un-reacted silver with $5 \%$ sodium thiosulphate.

Differentiation to chondrocyte: Passage-4 MSCs were subcultured consecutively in low-glucose DMEM with $15 \%$ FBS till passage- 7 cells with $90 \%$ confluence were seen. The adherent cells were trypsinized and cell viability was assessed. With $1 \times 10^{5}$ viable cells per $\mathrm{cm}^{2}$ and single cell suspension, a micromass culture was generated by seeding 5 -microliter droplets of cell solution (containing $8 \times 10^{4}$ cells) in the center of a well within a 6-well plate. The micromass culture was cultivated for 2 hours under highhumidified condition in a $37^{\circ} \mathrm{C}$ incubator with $5 \% \mathrm{CO}_{2}$. StemPro chondrogenesis differentiation media (GIBCO) was added. The media was serum-free and low-glucose. The media was supplemented every third day until the 21 st day. After that, chondrogenic pellet was stained using 1\% Alcian blue solution.

\section{Immuophenotyping}

MSCs were analyzed for cell surface marker expression at passage- 4 and passage- 7 . The cells were trypsinized, washed with staining buffer and incubated $\left(1 \mathrm{X} 10^{5}\right.$ cells in $\left.100 \mu \mathrm{L}\right)$ with combination of antibodies $(10 \mu \mathrm{L}$ each) for 45 minutes in the dark at $4^{\circ} \mathrm{C}$. The antibodies used were CFS-anti-rat-CD105, PE-antirat-CD29, APC-anti-rat-Sca-1 and PerCP-anti-rat-CD45. All antibodies were purchased from R\&D Systems, USA. Analysis was done in BD Acuuri C6 flow cytometer using CFlow Plus software. Isotype-matched antibodies were used as controls to determine non-specific staining. 


\section{Results and Discussion}

\section{Cell Cultures}

The cultures were observed daily by using a phase contrast inverted microscope to examine the morphology of the adherent cells. Primary culture from the bone chips showed some cells with circular shape and some with spindle shape with two processes on each side (Figure. 1a). During passage-1 and 2, in about four days' time after trypsinization and establishment of next passage of culture, individual adherent cells occupied about $90 \%$ of the surface of the culture flask (Figure. 1b). Remaining cells in the flask were floating and circular in shape. The adherent cells looked like fibroblasts with elongated cell bodies and long processes. From passage- 3 onwards, the size of the adherent cells increased and the cell body appeared either triangular or polyhedral with long processes and central nucleus. The amount of floating cells was also reduced. We propose that the adherent cells were MSCs because they exhibited the capacity to proliferate extensively in subsequent cultures until passage-12 and differentiate into adipocyte, osteoblast and chondrocyte.

\section{Proliferation of the Adherent Cell Population}

The adherent cells showed active proliferation in subsequent subcultures and the number of viable cells observed with the Trypan blue exclusion assay in passage- 5 was double to the number of cells plated in first primary culture (passage 0 ) with plating density of 2000 cells $/ \mathrm{cm}^{2}$ (Figure. 2). The proliferation of the cells was comparatively more in passage- 5 with primary plating density of 500 cells $/ \mathrm{cm}^{2}$ (nearly 5 -times as shown in sample M3, Figure 2). The rate of proliferation slowed down when the cultivated cells reached passage- 7 , but it was possible to cultivate the cells until passage -12 (Figure1, Figure2).

\section{Differentiation to adipocyte}

The treatment of the MSCs in passage- 4 with the adipogenic stimulatory supplement resulted in the differentiation of MSCs into adipocytes. Small lipid droplets appeared within the cytoplasm of a few differentiated cells, on the day 7 after addition of the adipogenic stimulatory supplement and occupied the whole cytoplasm of most of the cells, after 4 weeks. Lipid droplets within the cytoplasm of the differentiated cells were observed under oil red O staining (Figure. 3A 2) and compared with the control (Figure.3A 1).

\section{Differentiation to osteoblast}

Passage-4 MSCs when cultured in the osteogenic stimulatory supplement, differentiated into the osteoblast cells which started accumulating calcium in the extracellular matrix. The deposits were perceptible on the seventh day of culture. At $4^{\text {th }}$ week, the larger areas of calcification nodules turned dark-brown, with von Kossa staining (Figure. 3B 2).

\section{Differentiation to chondrocyte}

To induce chondrogenic differentiation, adherent cells of passage- 7 were cultured under hypoxic and serum-free conditions in pellet form. After 21 days, when the pellet was stained with Alcian blue, blue stained areas in between the cells indicated synthesis of proteoglycans (Figure. 3C 2). However the blue-stained areas were observed only towards the edge of the pellet indicating reduced chondrogenic differentiation of the MSCs cultivated in this study. Previous study has observed that the selection of CD105 positive MSCs favors chondrogenesis [16]. CD105 is an accessory protein of TGF- $\beta$ super family kinase receptor complexes. TGF- $\beta 3$ is the key transcription factor for differentiating MSCs into chondrocytes [17]. In this study, only a small proportion of the cultured MSCs expressed CD105, suggesting that this could be a potential reason for the reduced chondrogenic differentiation observed in this study (Figure 4) (Figure3).

\section{Phenotypic characterization}

Flow cytometry analysis revealed that more than $98 \%$ of the MSCs expressed Sca-1 and more than $63 \%$ of the MSCs expressed CD29 (Figure 4). Both Sca-1 and CD29 have been reported as surface-markers for mouse MSCs. Absence of CD45 positive cells $(0.6 \%)$ indicated that the culture was devoid of the cells of hematopoietic origin. A small percentage $(9.8 \%)$ of the cultured cells was positive for CD105. The origin of the cultured cells was from the marrow-free compact bone chips. Previous studies have linked the absence of CD105 expression with identification of MSCs having increased osteogenic gene expression $[18,19]$. Lower percentage of cultured MSCs expressing CD105 phenotype observed in this study can be extrapolated to the ori-

Figure 1. Morphological features of cultured cells from collagenase digested murine compact bone chips. (a) At 48 hours after initial culture, both fibroblast-like cells and round cells sprouted out from the bone fragments. (b) At 4th day after subculture, adherent cells with fibroblast-like morphology occupied $90 \%$ of the surface of the flask.
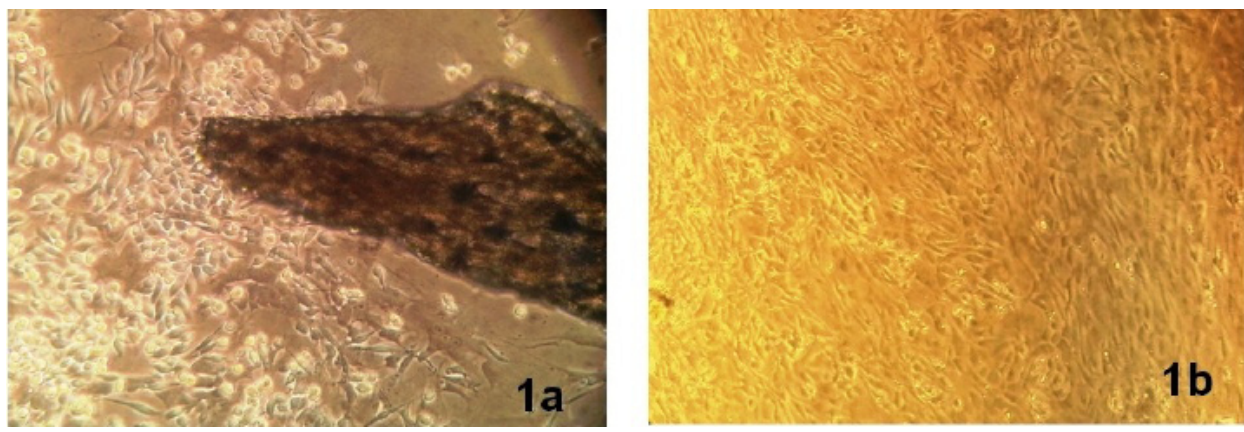
Figure 2. Bar chart showing the count of viable cells observed under Trypan blue exclusion assay during subsequent subculture from passage-0 (P0) to passage-5 (P5). The compact bone culture sample established from individual mouse is labelled as M1, M2, M3 and M4.

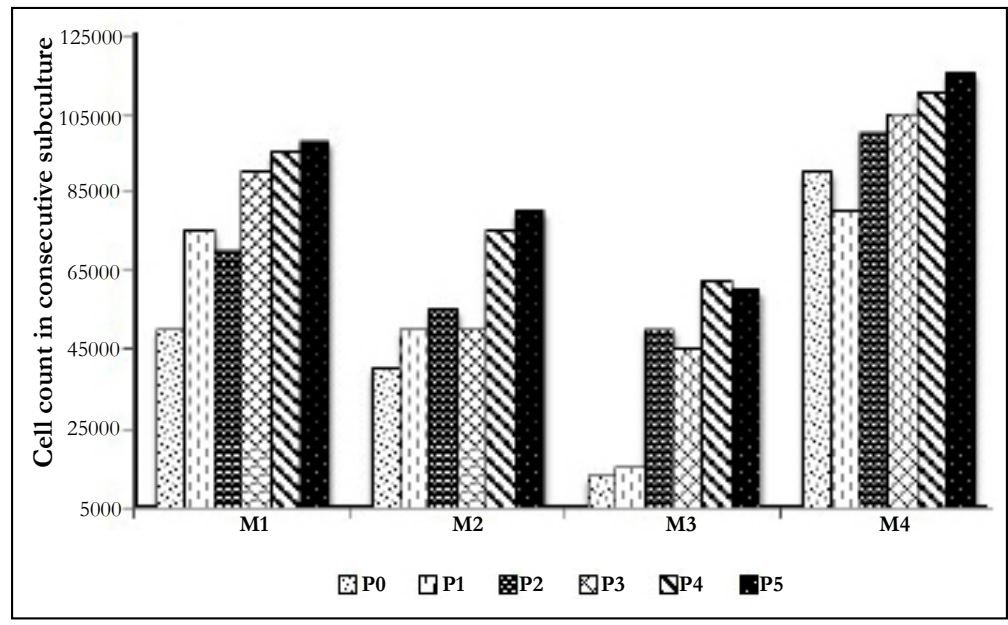

Figure 3. Phase contrast photomicroscopy of multilineage differentiation of the mesenchymal stem cells derived from murine compact bone chips culture. (3A 1) control undifferentiated MSC culture and (3A 2) Fat droplets in the differentiated adipocytes stained red (20x, Oil Red $O$ stain). (3B 1) control undifferentiated MSC culture and (3B 2) dark-brown calcium salt deposits in the extracellular matrix of differentiated osteoblast cells (20X, von Kossa stain). (3C 1) peripheral part of pellet of control undifferentiated MSC culture and (3C 2) bluish stained proteoglycans (red arrow) in between differentiated chondrocytes (10X, Alcian blue stain); Bar $50 \mu \mathrm{m}$.
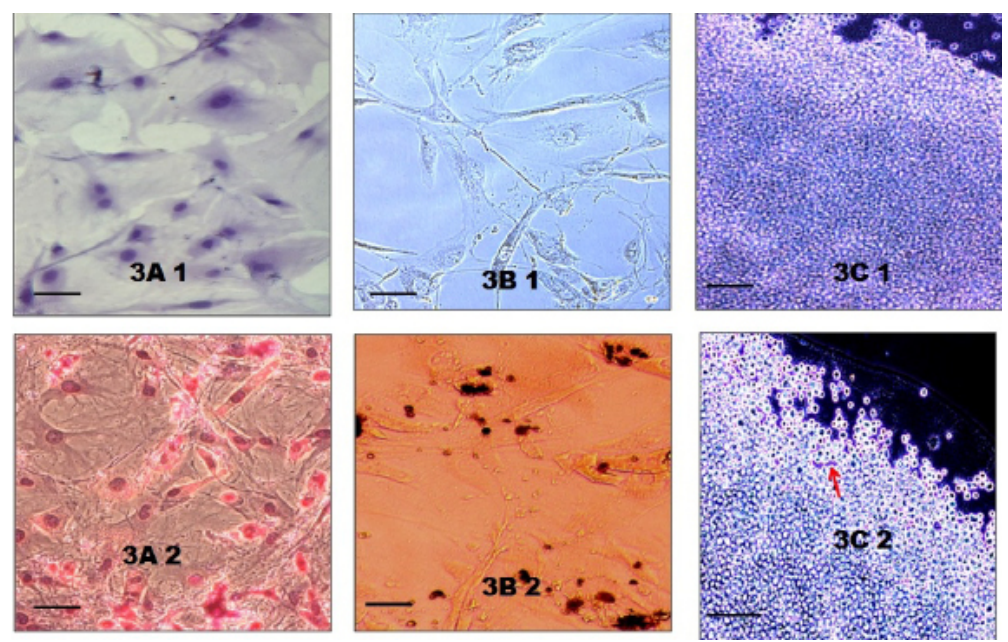

Figure 4. Flow cytometry analysis for phenotypic characterization of compact bone derived murine MSCs. The histogram with red lines shows isotype controls and histogram with gray line shows reactivity with indicated antibodies. Majority of cells were positive for Sca-1(98.2\%) and CD29 (63.4\%). Only a small subpopulation of cells $(9.8 \%)$ was positive for CD105. Cultured MSCs showed lack of CD45 expression.

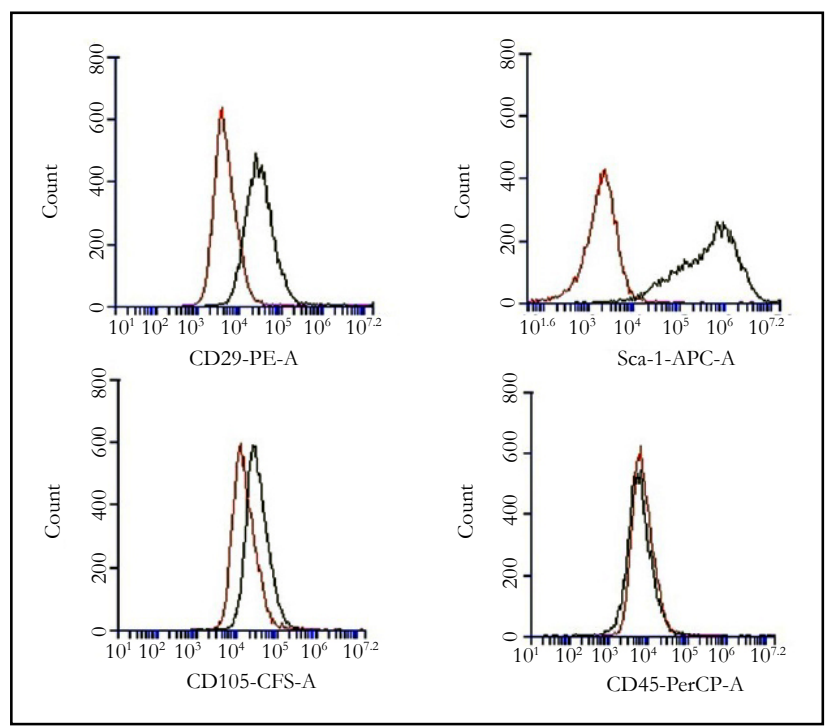


gin of the cultivated cells from the marrow-free compact bone (Figure4).

\section{Compact bone-derived mouse MSC and CD105 phenotype}

Compact-bone culture can yield good source of the mouse MSCs with relatively less capacity in multi-lineage differentiation after passage-10. Zhu et al., (2010) proposed a protocol that could yield a homogeneous population of mouse MSCs within a short period of time [20]. Present study followed the protocol proposed by Zhu et al. However compared to Zhu et al. study, which had reported $95 \%$ of the MSCs expressing CD105, this study, observed only $9.8 \%$ expression of CD105. The use of low-glucose DMEM and lack of specific amino acids like ribonucleosides and ribonucleotides might have prevented expression of CD 105. In a similar study of compact-bone derived mouse MSCs, Guo et al., (2006) observed that the proportion of CD105 cells decreased progressively in subsequent subcultures [21]. In a previous study, CD105-negative subpopulations of MSCs derived from the synovial membrane failed to form prominent pellet in chondrogenic differentiation media [22]. Anderson et al., (2013) proposed to redefine CD105 expression of mouse MSC and showed that CD105-negative cells suppressed the proliferation of CD4+ T cells more efficiently compared to CD105 positive cells [23]. MSCs cultured from the compact bone showed lack of CD105 expression in this study. Compact-bone derived MSCs can be attributed with promising experimental study value due to their unique surface antigen expression and lack of contamination with haematopoietic cells. This characterization was essential for the second part of our study in which we are planning to use these cells in vivo to restrore locomotion in mouse model of experimental autoimmune encephalomyelitis.

\section{Conclusion}

In this study, culture of the collagenase-digested compact bones from young 4-week-old C57BL/6 mouse showed presence of MSCs with significant proliferation capacity. The cells were characterized by prominent expression of surface markers, Sca1 and CD29, and relatively little expression of CD105, in flow cytometry. Lack of CD45 surface marker indicated absence of hematopoietic cells. These cultured cells displayed the ability to differentiate into osteoblast and adipocyte lineages when stimulated in the appropriate differentiating conditions. However, lack of prominent chondrocytic differentiation of the cultured cells, was extrapolated to be due to the relatively little populations of cells expressing CD105.

\section{Acknowledgement}

The study was supported by the TRGS Grant from the Centre for Research and Development of Taylor's University, Malaysia. The animal facility of the Brain Research Institute of Monash University Sunway Campus, Malyasia was used for the maintenance of the animals.

\section{References}

[1]. Friedenstein AJ, Chailakhjan RK, Lalykina KS (1970) The development of fibroblast colonies in monolayer cultures of guinea-pig bone marrow and spleen cells. Cell Tissue Kinet 3: 393-403.

[2]. Bianco P, Robey PG, Simmons PJ (2008) Mesenchymal stem cells: revisiting history, concepts, and assays. Cell Stem Cell 2: 313-319.
[3]. Peister A, Mellad JA, Larson BL, Hall BM, Gibson LF, et al. (2004) Adult stem cells from bone marrow (MSCs) isolated from different strains of inbred mice vary in surface epitope, rates of proliferation, and differentiation potential. Blood 103: 1662-1668.

[4]. Javazon EH, Colter DC, Schwarz EJ, Prockop DJ (2001) Rat marrow stromal cells are more sensitive to plating density and expand more rapidly from single-cell-derived colonies than human marrow stromal cells. Stem Cells 19: 219-225.

[5]. Xia CS, Zuo AJ, Wang CY, Wang YZ (2013) Isolation of rabbit bone marrow mesenchymal stem cells using density gradient centrifugation and adherence screening methods. Minerva Med 104: 519-525.

[6]. Izadpanah R, Trygg C, Patel B, Kriedt C, Dufour J, et al. (2006) Biologic properties of mesenchymal stem cells derived from bone marrow and adipose tissue. J Cell Biochem 99: 1285-1297.

[7]. Colter DC, Sekiya I, Prockop DJ (2001) Identification of a subpopulation of rapidly self-renewing and multipotential adult stem cells in colonies of human marrow stromal cells. Proc Natl Acad Sci USA 98: 7841-7845.

[8]. Boxall, SA, Jones E (2012) Markers for characterization of bone marrow multipotential stromal cells. Stem Cells Int 2012.

[9]. Colter DC, Class R, DiGirolamo CM, Prockop DJ (2000) Rapid expansion of recycling stem cells in cultures of plastic-adherent cells from human bone marrow. Proc Natl Acad Sci U S A 97: 3213-3218.

[10]. Pittenger MF, Mackay AM, Beck SC, Jaiswal RK, Douglas R, et al. (1999) Multilineage potential of adult human mesenchymal stem cells. Science 284: 143-147.

[11]. Alsalameh S, Amin R, Gemba T, Lotz M (2004) Identification of mesenchymal progenitor cells in normal and osteoarthritic human articular cartilage. Arthritis Rheum 50: 1522-1532.

[12]. Phinney DG, Kopen G, Isaacson R L, Prockop DJ (1999) Plastic adherent stromal cells from the bone marrow of commonly used strains of inbred mice: Variations in yield, growth, and differentiation. J Cell Biochem 72: 570-585.

[13]. Short B, Wagey R (2013) Isolation and culture of mesenchymal stem cells from mouse compact bone. In: Helgason CD, Miller CL, editor. Basic Cell Culture Protocols. Totowa NJ: Humana Press 335-347

[14]. Sung JH, Yang HM, Park JB, Choi GS, Joh JW, et al. (2008) Isolation and characterization of mouse mesenchymal stem cells. Transplant Proc 40: 2649-2654.

[15]. Yameen Z, Leavesley D, Upton Z, Xiao Y (2009) Multilineage differentiation potential of bone and cartilage cells derived from explant culture. Open Stem Cell J 1: 10-19.

[16]. Chang CB, Han SA, Kim EM, Lee S, Seong SC, et al. (2013) Chondrogenic potentials of human synovium-derived cells sorted by specific surface markers. Osteoarthr Cartilage 21: 190-199.

[17]. Bellón T, Corbí A, Lastres P, Calés C, Cebrián M, et al.(1993) Identification and expression of two forms of the human transforming growth factor-betabinding protein endoglin with distinct cytoplasmic regions. Eur J Immunol 23: 2340-2345.

[18]. Rosu-Myles M, Fair J, Pearce N, Mehic J (2010) Non-multipotent stroma inhibit the proliferation and differentiation of mesenchymal stromal cells in vitro. Cytotherapy 12: 818-830.

[19]. Levi B, Wan DC, Glotzbach JP, Hyun J, Januszyk M, et al. (2011) CD105 protein depletion enhances human adipose-derived stromal cell osteogenesis through reduction of transforming growth factor $\beta 1$ (TGF- $\beta 1$ ) signaling. J Biol Chem 286: 39497-39509.

[20]. Zhu H, Guo ZK, Jiang XX, Li H, Wang XY, et al. (2010) A protocol for isolation and culture of mesenchymal stem cells from mouse compact bone. Nat Protoc 5: 550-560.

[21]. Guo Z, Li H, Li X, Yu X, Wang H, et al. (2006) In vitro characteristics and in vivo immunosuppressive activity of compact bone-derived murine mesenchymal progenitor cells. Stem Cells 24: 992-1000.

[22]. Arufe MC, De la Fuente A, Fuentes-Boquete I, De Toro FJ, et al. (2009) Differentiation of synovial CD-105(+) human mesenchymal stem cells into chondrocyte-like cells through spheroid formation. J Cell Biochem 108: 145-155.

[23]. Anderson P, Carrillo-Gálvez AB, García-Pérez A, Cobo M, et al. (2013) CD105 (endoglin)-negative murine mesenchymal stromal cells define a new multipotent subpopulation with distinct differentiation and immunomodulatory capacities. PLoS One 8: e76979. 